\title{
Binary metal-doped methoxide catalyst for biodiesel production from palm stearin
}

\begin{abstract}
Heterogeneous transesterification of vegetable oils offers an environmentally more attractive option for biodiesel production compared with the conventional homogeneous processes. Thus, double metallic methoxide catalyst was developed in the present study, aiming to improve the transesterification of low-cost palm stearin (PS) and reduce waste generation. The physicochemical properties of the synthesized catalyst were studied by various techniques such as X-ray diffraction, field-emission scanning electron microscopy, temperature-programmed desorption-CO2 coupled with mass spectrometry, and Brunauerï Emmettï Teller surface area analyses. The optimum parameters were obtained via the response surface methodology coupled with a central composite design. Transesterification with the highest biodiesel yield of $98 \%$ was obtained using $3 \%$ catalyst loading, methanolto-PS ratio of $11: 1,125$ min reaction time, and $70^{\circ} \mathrm{C}$ temperature. This catalyst appears to be a promising candidate to replace homogeneous catalysts for biodiesel production, as it required short reaction duration and offered high reusability.
\end{abstract}

Keyword: Biodiesel; Calcium methoxide; Catalyst; Zirconia support 